\section{An Automatic Staining Machine Designed for Staining Blood Films Made on Coverslips}

\author{
WILLIAM M. DAVIDSON. F. R. BAREHAM. \\ L. D. KITCHEN AND S. H. PEGG
}

From the Clinical Pathology Department and Physics Laboratory, King's College Hospitel and Medical School, Denmark Hill, London

(RFCEIVED FOR PUBLICATION FEBRUARY 6, 1957)

In most forms of microscopical investigation some form of routine staining is essential. Many such procedures are merely repetitive yet are time consuming. A machine to lift the preparations from staining pot to staining pot at predetermined intervals is not a luxury but a basic requirement in a laboratory where a simple staining method is used 50 or more times a day.

Originally conceived to relieve the laboratory staff of unnecessary routine work, the present machine was expected to produce staining results of reasonable quality. The standard achieved has been far beyond expectation and for the past six years many thousands of films have been stained to a uniform standard, otherwise attained only by the personal care of an experienced technician.

In the machine a central shaft carries 12 radial arms. The ends of these arms come to rest over the centre of each reagent pot. The blood smears are made on $\frac{7}{8}$ in. square No. 2 coverslips, by sliding apart two of these after a drop of blood between them has been allowed to spread almost to its full extent by capillary attraction (Ehrlich and Lazarus, 1900 ; Wintrobe, 1956). After drying, the coverslips are placed in perspex containers which are attached to swivel hooks at the ends of the arms. Loose perspex discs above the hooks cover the pots during the time of immersion to reduce evaporation. At predetermined intervals the central shaft is raised by an electric motor until the slide carriers are clear of the pots. At this point a second motor is brought into action and rotates the arms in a counter-clockwise direction until each is above the next "station." The first motor then takes over again and lowers the carriers into the solutions. the whole movement being completed in under 20 seconds. The staining period is determined by an electronic timer which can be set at intervals between a half and three minutes. In this way the preparations are passed through the successive solutions and are finally removed by striking a ramp at "station twelve." Apart from loading the carrier and attaching it to the hook at "station one" the process is entirely automatic, and as arranged the machine has a maximum staining capacity of 120 coverslips hourly.

A range of immersion times, from a half to over 12 minutes, can be predetermined by varying the setting of the timer and the number of pots used for any reagent. In this laboratory the Jenner-Giemsa method is in routine use for the staining of human and animal blood films. The reagents are contained in standard ointment pots with an internal diameter of $6 \mathrm{~cm}$., a depth of $4 \mathrm{~cm}$. and a capacity of $80 \mathrm{ml}$. The timer is set at two minutes. Atsolute methyl alcohol in two pots gives four minutes for fixing. Three pots of Jenner's stain, diluted in an equal quantity of $p \mathrm{H} 6.8$ phosphate buffer, give optimum staining of the red cells and the cytoplasm of the leucocytes; and a further six minutes in Giemsa stain, diluted 1 in 10 in $p \mathrm{H} 6.8$ phosphate buffer, enhances the staining of the chromatin of the nuclei and the granules in the neutrophils and platelets. Finally, two pots of buffer solution, aerated to keep up a constant washing action remove any scum which has formed. After passing through the buffer, the films in their carrier strike the ramp and come off the machine. They are normally allowed to dry in air, but a quick rinse in distilled water, followed by blotting dry, may improve the final differentiation of the structural details.

In staining coverslip spreads made from tiny fragments of bone marrow, the longer time necessary ( 12 minutes) is achieved by sending the preparations twice through the Giemsa stain pots.

With the reagents detailed it costs 1 s. $6 \mathrm{~d}$. to load this machine against approximately $1 \mathrm{~s}$. for a hand method using four Coplin jars.

Although this machine, as originally developed and constructed by the Physics Laboratory of King's College Hospital, has worked satisfactorily for over six years, it has long been evident that it could be simplified. It contains two electric motors, several relays and many other electrical components, in addition to the mechanical parts for the lifting and turning. Further, the two motors require perfect synchronization and have to be provided with a braking mechanism for efficient operation. In a newly designed modification mechanical and electrical simplification has been achieved. Only one motor is required to operate the lifting and turning mechanisms which are mechanically linked and cannot fall out of synchronism. After designing the basis of the simplified machine and producing a working model of the mechanism, it seemed unwise to incur further development costs and the idea has been passed to a commercial firm (Elliotts Liverpool, Ltd.).

This mechanical aid to staining has proved of the $N$ greatest value in the laboratory routine. The uniformly or high quality of the staining attained has made it possible $N$ to place greater weight upon fine changes in the details $\underset{\mathrm{W}}{\mathrm{N}}$ of the cell morphology.

\section{Stains Used}

1. Jenner and Giemsa.

2. Buffer $p \mathrm{H} 6.8$ in form of phosphate buffer tablets.

3. Methyl alcohol (maximum acetone $0.05 \%$ ).

4. Glycerine B.P.

Jenner is used as a $0.5 \%$ alcoholic solution, and $\stackrel{\mathbb{D}}{\mathbb{D}}$ Giemsa as a $0.76^{\circ} \%$ solution in equal parts of glycerine and alcohol. 


\section{Summary}

An automatic machine for the staining of microscopical preparations is described. This machine, built in the hospital, has been in successful routine use for six years. Future developments are indicated.

Designed originally to save time and labour, it has proved of even greater worth in the uniformly high quality of the staining achieved.

Such a machine is a necessity in a busy laboratory where one staining method is used many times a day.

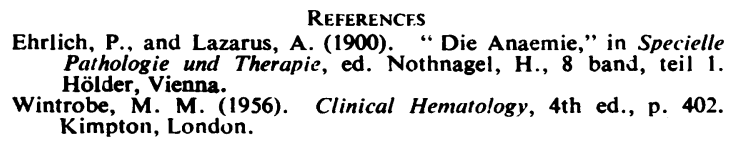

\section{An Apparatus for Holding Chromatography Paper During "Spotting-On" \\ D. N. BARON}

From the Department of Chemical Pathology, the Royal Free Hospital, London, W.C.I

(RECEIVED FOR PUBLICATION MARCH 19, 1957)

It is often difficult to find a convenient support for the paper when applying the spots of material before chromatography.

The apparatus described here has been in constant use for the past two years. It keeps chromatography paper clean and still during "spotting-on," and also holds it so that a drying current of air or nitrogen can, if required, be continuously applied from above or below.

It consists of two $27 \mathrm{~cm}$. square plates of $\frac{1}{4}$ in. perspex. One sector, $21 \mathrm{~cm} . \times 3 \mathrm{~cm}$., is cut out of each plate in the same position, $3 \mathrm{~cm}$. from one edge. Four L-section legs, $5 \mathrm{~cm}$. long, made from $\frac{1}{8}$ in. perspex, are glued to one plate (Fig. 1). The cost of the perspex is about 12s. 6d., and the apparatus takes a skilled technician about three hours to make.

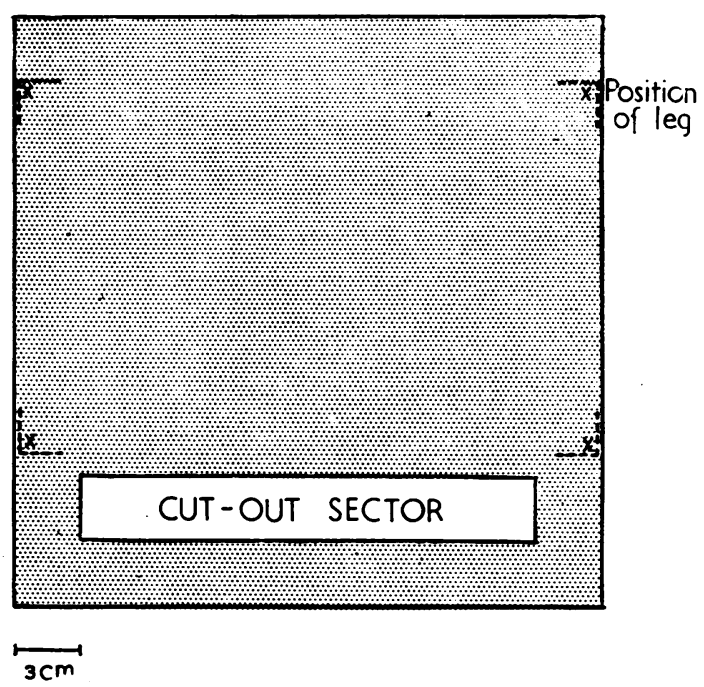

Fig. 1._Plan of perspex plate (for dimensions see text).

In use the lower plate stands horizontally on its legs. A standard 10 in. square paper is placed symmetrically on it, and the second plate is placed over the paper so that one cut-out sector is above the other, the paper being sandwiched between the 